\title{
Zwischen übersteigerter und fehlender Solidarität. Die Covid-19-Pandemie aus biopolitischer Perspektive nach Foucault
}

Willibald J. Stronegger

«Desormais, la sécurité est au-dessus des lois »
Michel Foucault, $1977^{1}$

\section{Einleitung}

In einer Zeit ohne metaphysische Realitäten und Begriffe treten bedrohliche Epidemien nicht mehr als Konsequenz für kollektiv begangene Verfehlungen, den Bruch alter Rechte oder die Missachtung eines göttlichen Willens in Erscheinung. Es begleitet sie kein ritualisiertes Beziehungsgeschehen, sie folgen nicht auf eine kollektive Untreue zu einem verborgenen Gott oder Seinsgrund. Sie sind heute eine ausschließlich bio-psychosoziale Bedrohung, die nach effektiven und effizienten bio-psycho-sozialen Maßnahmen auf wissenschaftlicher Grundlage verlangt. Die Covid-19Pandemie trifft im Jahre 2020 auf Gesellschaften, für die gefährliche Pandemien keine historische, regelmäßig wiederkehrende Normalität mehr sind, wie sie es bis zu den Weltkriegen auch in den Industrienationen waren. Seither haben die modernen Staaten eine biopolitische Transformation (im Sinne Michel Foucaults Konzept) erfahren, welche die Frage nach dem politischen Umgang mit Pandemien neu aufwirft - nämlich gerade die Frage nach der Rolle biopolitischer Rationalität im staatlichen Verhalten angesichts hygienischer Massenbedrohungen der Art, wie es die Covid-19-Pandemie des Jahres 2020 ist.

Der Beitrag stellt die These vor, dass natürliche Massenbedrohungen den Anlass zu einer Verstärkung bzw. Beschleunigung jenes biopolitischen Transformationsprozesses geben können, in welchem sich die Industriegesellschaften insbesondere seit dem 18. Jahrhundert nach den Analysen des

1 Dt.: „Von nun an steht die Sicherheit über den Gesetzen“; Foucault, Dits, 366. 
französischen Philosophen Michel Foucault befinden. ${ }^{2}$ Diese Verstärkung betrifft zwei auf den ersten Blick konträre Ansatzpunkte und Wirkungen. Der erste Punkt betrifft die verstärkte Legitimierung und Umsetzung von Umverteilungs- und Solidaritätsmaßnahmen, der zweite eine gesteigerte Entsolidarisierung gegenüber vulnerablen und alten Bevölkerungsteilen. Es kommt nach der im Folgenden vorgestellten These potentiell zur verstärkten Ausbildung einer bestimmten Form von Humanität bzw. Solidarität, die für (idealtypische) biopolitische Staaten charakteristisch ist, bei möglicherweise gleichzeitigem Abbau von Schutz für die Gefährdetsten, gemäß der Foucault'schen Formel der Biopolitik als „Lebenmachen und Sterbenlassen" (le droit de faire vivre et de laisser mourir). ${ }^{3}$ Beide Effekte sind im Grunde einfach die durch die Gunst der Stunde genützte verstärkte Anwendung bestehender biopolitischer (bzw. gouvernementaler) Haupttaktiken der staatlich-institutionellen Transformationen. Um den Argumentationskreis zu schließen, beginnt der Beitrag mit einer Skizze dessen, worin die biopolitische Transformation der Gesellschaft besteht, gefolgt von einer kurzen Darstellung ihrer Haupttaktiken und -techniken, deren ursprüngliche Legitimation auch in biologischen Bedrohungen der Bevölkerung zu verorten ist.

\section{2. ... d'augmenter leur santé: ${ }^{4}$ Die Transformation des Individuums und des} Staates

„Wir leben im Zeitalter der Gouvernementalität, die im 18. Jahrhundert entdeckt wurde. ${ }^{\text {"5 }}$ Michel Foucault, 1978

Michel Foucault hat in seinen bekannten Machtanalysen der 1970er Jahre die Transformation der institutionellen und öffentlich-staatlichen Strukturen und der Regierungstechniken seit dem 17. Jahrhundert als Ausdruck einer Biomacht (bio-pouvoir) bzw. Biopolitik (bio-politique) gedeutet, als ein umfassendes Phänomen, welches in einer „allgemeinen Medikalisierung des Daseins" besteht:

2 Vgl. Stronegger, Legitimität.

3 Foucault, Société, 214.

4 Dt.: „... ihre Gesundheit zu mehren“; Foucault, Sicherheit, 158.

5 Foucault, Sicherheit, 164. 
Ganz schematisch könnte ich sagen, das große Problem der abendländischen Gesellschaften vom Mittelalter bis ins 18. Jahrhundert war das Recht, das Gesetz, die legitime und gesetzliche Herrschaft. In all den politischen Kämpfen, die Europa bis ins 19. Jahrhundert erschütterten, bemühte man sich, eine Gesellschaft des Rechts zu schaffen und die Rechte des Einzelnen zu sichern. Und genau in dem Moment als man glaubte, als z.B. die französischen Revolutionäre glaubten, eine Gesellschaft des Rechts verwirklicht zu haben, da geschah etwas anderes: etwas, das zum Eintritt in eine Gesellschaft der Norm, der Gesundheit, der Medizin, der Normalisierung führte, welche jetzt die wesentliche Funktionsweise ist. Und genau diesen Vorgang habe ich zu analysieren versucht. ${ }^{6}$

Im Rahmen seiner Machtanalysen bringt er in einem Gespräch eine Beobachtung zur Sprache, die zur Skepsis gegenüber dem Funktionieren der modernen Normalisierungsgesellschaften Anlass gibt:

Genau zu dem Zeitpunkt, als der Staat seine größten Massaker in Angriff nahm, begann er auch, sich um die körperliche und geistige Gesundheit des Einzelnen zu sorgen. [...] Dieses Spiel zwischen dem Leben und dem Tod ist eine der Hauptparadoxien des modernen Staates. $^{7}$

Die Überlegungen zur Biopolitik entwickelte Foucault über einen längeren Zeitraum, auch wenn er diesen Begriff erst in den Jahren 1975/76 einführte und verwendete. Die Ursprünge des Konzepts liegen in seinen früheren Arbeiten, insbesondere im Werk Überwachen und Strafen, und in den Analysen zu den politischen Reglements im Zusammenhang mit den großen historischen Epidemien wie Lepra, Pest und Pocken. Mit der Einführung des Begriffs der Biopolitik entsteht ein eigenständiges, d. h. von konkreten epidemiologischen Ereignissen unabhängiges Konzept zur Machtund Regierungspraxis moderner Staaten, das jedoch einer Weiterentwicklung unterlag, vor allem durch die fortschreitende Einbeziehung der historischen Entwicklung der politischen Systeme in der Neuzeit. Zuletzt, in seinen Analysen liberaler Regierungsrationalitäten und des homo oeconomicus, verliert der Begriff der Biopolitik in seinen Arbeiten an Bedeutung

6 Foucault 1977 in einem Gespräch mit M. Osorio über das „medizinische Denken“; Foucault, Dits, 373 u. Schriften, 483.

7 Foucault, Dits, 802. Foucault nimmt in diesem Zusammenhang Bezug auf die Napoleonischen Kriege und die Errichtung des Sozialismus in der Sowjetunion und in China. 
und geht in jenem der sogenannten ,gouvernementalen Vernunft“8 auf: „Wenn man also verstanden hat, was dieses Regierungssystem ist, das Liberalismus genannt wird, dann, so scheint mir, wird man auch begreifen können, was die Biopolitik ist".?

Was ist bei Foucault die "gouvernementale Vernunft“, die Gouvernementalität? Sie tritt in den sozialen und politischen Veränderungen des 18. Jahrhunderts in Erscheinung, im „Übergang von einem Regime, das durch die Strukturen der Souveränität beherrscht ist, zu einem Regime, das durch die Techniken des Regierens beherrscht ist [...]“, und der Übergang tritt „im Kontext der Bevölkerung ein und folglich im Kontext der Geburt der politischen Ökonomie“ ${ }^{10}$ denn die Bevölkerung als natürliche Gegebenheit (d.h. nicht als eine Menge juridischer Subjekte, sondern als natürliche Entität, die statistisch vermessen wird), tritt „als der höchste Zweck der Regierung zutage“. Was aber, so fragt Foucault weiter, mag nun im Grunde genommen der Zweck dieser Regierung der Bevölkerung sein? „Sicherlich nicht zu regieren, sondern das Geschick der Bevölkerungen zu verbessern, ihre Reichtümer, ihre Lebensdauer, ihre Gesundheit zu mehren. [...] Eher als die Macht des Souveräns erscheint die Bevölkerung also als Ziel und Instrument der Regierung." 11

Auf welche Weise funktioniert eine Regierung der Bevölkerung, die es also nicht primär mit Rechtssubjekten als ihren Zielobjekten zu tun hat, sondern mit den „natürlichen“ Zuständen der Menschen und ihrer bevölkerungsmäßigen Anhäufung? Ansatzpunkt einer solchen Regierung ist das „Interesse“: „Das Interesse als Bewusstsein jedes einzelnen Individuums, das mit den übrigen die Bevölkerung bildet, und das Interesse als Interesse der Bevölkerung [...] sind die Zielscheibe und das Hauptinstrument der Regierung der Bevölkerungen. Die Geburt einer Kunst oder jedenfalls die Geburt völlig neuer Taktiken und Techniken“. ${ }^{12}$ Diese Regierungstechniken der Bevölkerung erfordern zur rationalen Ausübung die Bildung eines neuartigen Wissens über alle die Vorgänge, die im realen Leben in den Be-

8 Der Begriff Gouvernementalität ist die deutsche Form des frz. „gouvernementalitéc, letzteres abgeleitet aus „gouvernemental“ (wie „spatialité" aus „spatial“); vgl. Foucault, Geburt, 482. Der Begriff dient bei seiner Einführung in der 4. Sitzung der Vorlesung 1978 dazu, „das im 18. Jahrhundert installierte Machtsystem zu benennen, das ,als Hauptzielscheibe die Bevölkerung, als wichtigste Wissensform die politische Ökonomie und als wesentliches technisches Instrument die Sicherheitsdispositive hat"“, Foucault, Geburt, 482.

9 Foucault, Geburt, 43.

10 Foucault, Sicherheit, 159.

11 Foucault Sicherheit, 158.

12 Foucault, Sicherheit, $158 \mathrm{f}$. 
völkerungen ablaufen: „nämlich über genau das, was man ,Ökonomie“ nennt “. ${ }^{13}$ Mit dem Auftreten der Bevölkerung als eines neuen Subjekts (und Objekts) hatte sich die "politische Ökonomie“ als neue Wissenschaft bilden können, zugleich mit „dem Feld der Ökonomie und der Bevölkerung" als des neuen Interventionsbereichs des Regierens.

Einen Staat zu regieren wird also heißen, die Ökonomie anzuwenden, eine Ökonomie auf der Ebene des Staates als Ganzem, das heißt, man wird, was die Einwohner, die Reichtümer, das Verhalten aller und jedes einzelnen betrifft, eine Form von Überwachung und Kontrolle ausüben, die nicht weniger aufmerksam ist als die des Familienvaters über die Hausgemeinschaft und ihre Güter. ${ }^{14}$

Die ubiquitäre Überwachung und Kontrolle des Individuums entspringt bei Foucault einer auf den Einzelnen gerichteten Regierungstechnologie, welche er in Bezugnahme auf ihren Ursprung in den Seelsorgetechniken der christlichen Kirchen als christliches Pastorat oder Pastoralmacht bezeichnete. Die Hauptfunktion des Pastorats ist es, so wie ein "guter Hirte“, denen, „über die man wacht, Gutes zu tun“. Der Zweck dieser Form der Macht liegt darin, „die Individuen zu zwingen, ihre Arbeitsleistung, ihre Kräfte, ihre Fähigkeiten zu steigern, kurz, alles das zu erhöhen, was für ihre Verwendung im Produktionsapparat der Gesellschaft notwendig war" ${ }^{15}$ Anders als im juristischen Denken, das mit den Kategorien des Erlaubten, Verbotenen, und dem Instrument der Strafe arbeitet, geht es hier um die Unterscheidung von „normal“ und „anomal“ und um „Besserungsinstrumente“ (moyens de correction) zur "Transformation des Individuums“ und um „eine damit in Verbindung stehende ganze Technologie menschlichen Verhaltens. " 16 Neben der Bevölkerung und der wissensmäßigen Abbildung ihrer kollektiven Eigenschaften mittels Statistiken und Indikatoren entsteht zusätzlich der komplementäre Pol eines „personalisierten“ Wissens auf der Ebene des Einzelnen und seiner Fähigkeiten bzw. „Capabilities":17

13 Foucault, Sicherheit, 159.

14 Foucault, Sicherheit, 144.

15 Foucault, Schriften, 716.

16 Foucault, Dits, 374.

17 Charakteristisch ist das „transformierte“ Selbstverständnis des modernen Menschen, das nicht mehr über Bindungen und Zugehörigkeiten geht, sondern über seine „Kompetenzen“ und "Capabilities“, siehe z. B. der Capability-Approach M. Nussbaums. Vgl. Nussbaum, Creating capabilities. 
Mit der Medikalisierung, der Normalisierung erhalten Sie gleichsam eine Hierarchie von Individuen mit mehr oder weniger großen Fähigkeiten. Der eine entspricht einer bestimmten Norm, der andere weicht davon ab; den einen kann man bessern, den anderen nicht; den einen kann man mit einem bestimmten Mittel bessern, beim anderen muss man andere Mittel einsetzen. Diese Einordnung der Individuen nach dem Grad ihrer Normalität ist meines Erachtens eines der großen Machtinstrumente der heutigen Gesellschaft [...] nach dem Grad der Produktivität im weitesten Sinne. ${ }^{18}$

Foucault bezeichnet das Dreieck von Regierung, Bevölkerung und politischer Ökonomie, das eine seit dem 18. Jahrhundert andauernde Verbindung realisiert, „die auch heute noch nicht aufgelöst ist“19, als „Gouvernementalität“. Diese ist zugleich Vorgang wie Ergebnis des Vorgangs, „durch den der mittelalterliche Staat der Gerichtsbarkeit, der im 15. und 16. Jahrhundert zum Verwaltungsstaat wurde, sich nach und nach ,gouvernementalisiert ${ }^{6}$ hat" ${ }^{\prime 20}$ „Das, was es für unsere Modernität, das heißt für unsere Aktualität an Wichtigem gibt, ist also nicht die Verstaatlichung der Gesellschaft, sondern das, was ich eher die ,Gouvernementalisierung' des Staates nennen würde". ${ }^{21}$ Es handelt sich hier für Foucault um einen fundamentalen Umbruch in der Ordnung der neuzeitlichen Gesellschaften, wie er ihn schon zuvor für das Erscheinen der Biopolitik diagnostizierte.

\subsection{Si Dieu n'existe pas ....22 Die Achillesferse des gouvernementalen Staates}

Zeitgleich mit dem Umbruch zur Gouvernementalisierung des Staates stellt sich ein Problem mit neuer und besonderer Schärfe: das Problem der Souveränität, d.h. die Frage, ,welche juristische Form, welche institutionelle Form, welche Rechtsgrundlage man der Souveränität, die einen Staat charakterisiert, würde geben können" ${ }^{23}$ Nochmals deutlicher formuliert: Wie kann Herrschaft, politische Autorität, wie können Recht und Gesetz legitimiert werden, wenn es kein ideelles oder metaphysisches Prinzip gibt, wenn alles nur "natürlich“ im modernen naturwissenschaftlichen

18 Foucault, Dits, 374f, u. Schriften, 485.

19 Foucault, Sicherheit, 162.

20 Foucault, Sicherheit, 163.

21 Foucault, Sicherheit, 163.

22 Dt. „Wenn Gott nicht existiert ...“; Foucault, Sicherheit, 355.

23 Foucault, Sicherheit, 160. 
Verständnis ist, die Menschen, die Bevölkerung, die Regierung, sowie auch die Natur selbst. „Entfernen Sie Gott aus dem System, und wenn Sie den Leuten sagen, dass man gehorchen muss und dass man einer Regierung gehorchen muss, in wessen Namen muss man dann gehorchen? Kein Gott mehr, keine Gesetze mehr. Kein Gott mehr, keine Verpflichtungen mehr. Und es gibt jemanden, der hat gesagt: ,Wenn Gott nicht existiert, ist alles erlaubt'. Dieser Jemand ist nicht derjenige, den Sie meinen. Es ist Hochwürden Contzen im Politicorum libri decem, im Livre des politiques, der von 1620 datiert $[\ldots]$... ${ }^{24}$

Eine rein naturalistische, d.h. säkulare Gesellschaftsordnung kann gewiss vorläufig vom geliehenen normativen Kapital übernommener (oder überkommener) Traditionen leben. Nach dem Böckenförde-Diktum der Staatsrechtslehre ist es fraglich, ob der säkulare Staat aus sich die Voraussetzungen herstellen kann, die ihn legitimieren und seine ihn tragende Freiheitlichkeit verbürgen können.

Bis zum 19. Jahrhundert war ja, in einer zunächst sakral, dann religiös gedeuteten Welt die Religion immer die tiefste Bindungskraft für die politische Ordnung und das staatliche Leben gewesen. Lässt sich Sittlichkeit innerweltlich, säkular begründen und erhalten, kann der Staat sich auf eine 'natürliche Moral' erbauen? Wenn nicht, kann er - unabhängig von dem allen - aus der Erfüllung der eudämonistischen Lebenserwartung seiner Bürger leben? Diese Fragen führen zurück auf eine tieferliegende, prinzipielle Frage: Wieweit können staatlich geeinte Völker allein aus der Gewährleistung der Freiheit des einzelnen leben ohne ein einigendes Band, das dieser Freiheit vorausliegt?. ${ }^{25}$

Die Antwort des gouvernementalisierten Staates besteht nun exakt in der von Böckenförde angesprochenen Alternative, nämlich die „Erfüllung der eudämonistischen Lebenserwartung seiner Bürger" zur Staatsaufgabe und zum höchsten gesellschaftlichen Ziel zu machen, und genau diesen Zwecken dienen die neuen Regierungstechniken. Zu diesen gouvernementalen Techniken gehören einerseits die auf das Individuum orientierte Pastoralmacht und andererseits die bevölkerungsbezogenen Verwaltungs- und Regierungstechniken der Policey ${ }^{26}$, die sich im 17. und 18. Jahrhundert, im

24 Foucault, Sicherheit, 355. Foucault nimmt Bezug auf den deutschen Jesuiten Adam Contzen (1571-1635) und sein zur Zeit des Dreissigjährigen Krieges erschienenes Hauptwerk.

25 Böckenförde, Entstehung, 59.

26 Die alte Orthografie wird verwendet, um den Begriff vom heutigen Verständnis des Begriffs Polizei erkennbar zu unterscheiden. 
frühmodernen merkantilistischen Verwaltungs- und Wirtschaftsstaat, in den Policeywissenschaften als einer Frühform biopolitischer Rationalität konkretisierten:

Die Polizei ist die Gesamtheit der Interventionen und Mittel, die sicherstellen, dass das Leben, das Etwas-mehr-als-nur-leben, das Zusammenleben tatsächlich zur Bildung und Steigerung der Kräfte des Staates nützlich sein wird. Wir haben also mit der Polizei einen Kreis, der im Ausgang vom Staat als rationale und berechnete Interventionsmacht über die Individuen zum Staat als Gesamtheit wachsender oder zu steigernder Kräfte zurückkehrt. Aber wodurch wird dieser Kreis hindurchgehen? [...] Der Kreis wird durch das Leben der Individuen hindurchgehen, aber wird auch durch ihr Etwas-mehr-als-nur-leben hindurchgehen, durch ihr Mehr-als-leben hindurchgehen, d.h. durch das, was man zu jener Zeit die Bequemlichkeit der Menschen, ihre Annehmlichkeit, oder auch ihre Glückseligkeit nennt [...] aus dem Glück der Menschen soll der Nutzen für den Staat entstehen [...] Man findet beispielsweise bei Delamare die Behauptung, dass der einzige Gegenstand der Polizei, darin besteht, den Menschen zur vollkommensten Glückseligkeit zu führen, die er in diesem Leben genießen kann' [...]. Alles, was von der bloßen Existenz zum Wohlbefinden [orig. bien-être] führt, alles, was zur Herstellung dieses Wohlbefindens über die bloße Existenz hinaus dient, und zwar auf solche Art und Weise, dass das Wohl der Individuen die Kraft des Staates ist, das scheint mir das Ziel der Polizei zu sein. ${ }^{27}$

Neben der bevölkerungsorientierten verwaltungstechnischen Seite, der Policey, ist die zweite, gewissermaßen subjektiv-individuelle Seite der Biopolitik, die Minderung des Leidens und die Steigerung des individuellen Wohlbefindens (bien-être), heute oft in die Begriffe Well-being oder Lebensqualität gefasst. Dieses „höchste Gut“ (das summum bonum) des gouvernementalen Staates ist etwas völlig anderes als jenes in der alten metaphysischen Tradition angestrebte, wie Foucault anmerkt: „Das ,Gut', das in der Definition der Regierung beim Hl. Thomas gemeint war (man soll es so einrichten, dass die Menschen sich wohl verhalten, um Zugang zum höchsten Gut zu erlangen), ändert seinen Sinn vollständig“28. Heute wäre wohl „bio-psycho-soziales Wohlbefinden“ (analog zur Gesundheitsdefiniti-

27 Foucault, Sicherheit, 469 f. U.a. hier wird deutlich, dass als „Leben“ in der Biopolitik Foucaults kein „nacktes Leben“ verstanden wird, wie zuweilen behauptet.

28 Foucault, Sicherheit, 472. 
on der WHO) die passende Bezeichnung für dieses angestrebte Glück naturalistischer Provenienz als vollendeter Ausdruck eines „Lebens in naturalistischer Fülle". Übertragen auf die gesellschaftliche Ebene handelt es sich um einen Kampf im Namen der „Glückseligkeit im Sinne eines naturalistisch gedachten Wohlbefindens, Förderung des Glücks im Hinblick auf soziale Wohlfahrt, Glück als die jeweils erreichbare größtmögliche Bedürfnisbefriedigung" ${ }^{29}$. Dieser sogenannte Sozial- bzw. Masseneudämonismus „prägt die Wertorientierung des modernen Menschen. Mit Nachdruck verlangt die Förderung der Wohlfahrt aller nach normativer Geltung und drängt auf Verwirklichung " ${ }^{\text {"30 }}$, gerade auch auf der strukturellen Ebene des Staates und der Regierungstechniken. Die Aufgabe des naturalistischen Wohlfahrtsstaates ist die „Idee der Minderung des Leidens und der Stärkung der Wohlfahrt, das Prinzip des größten Glücks der größten Zahl“ (vgl. die utilitaristischen Konzepte von Jeremy Bentham und John Stuart Mill). Glückszuwachs muss quantifizierbar sein für eine gerechte, d. h. berechnete und geplante Vermittlung der Glücksgüter durch den Staat: „Bentham konstruiert einen Staat, der als Verteiler von Glücksquanten fungiert. "31 Die Entgrenzung der naturalistischen Optimierung realisiert sich jedoch nur über die vollständige Vermessung und Kontrolle des Einzelnen und der Bevölkerungen, über die vollständige Technisierung der sozialen Welt mittels einer umfassenden „Quantifizierung des Sozialen“32.

\section{2 „Bien-être“: Die raison d'être des gesellschaftlichen Zusammenhalts im gouvernementalen Staat}

Die Bestimmung des Menschen scheint in diesem biopolitischen Verständnis in der irdischen Glückseligkeit eines Lebens ohne Leid und Krankheit zu liegen, und alles Sozialgefüge und staatliche Unterfangen habe die Herstellung dieses Glücks als Wohlfahrt aller zur Aufgabe. Diese Auffassung begründet zugleich einen den modernen Staat leitenden, historisch neuen Begriff von Humanität:

Es scheint, dass heute die Ausrichtung auf das Glück im Sinne der Wohlfahrt aller als Inbegriff und Kriterium der Humanität auftritt. Das Prinzip des größten Glücks der größten Zahl wird zum letztgülti-

29 Delikostantis, Humanitarismus, 5.

30 Delikostantis, Humanitarismus, 6.

31 Schulz, Philosophie, 747.

32 Vgl. Mau, Das metrische Wir. 
gen Maßstab humanen Handelns erhoben. Allein die Orientierung an der allgemeinen Wohlfahrt scheint die Gesellschaft als eine humane zu qualifizieren. ${ }^{33}$

„Was bleibt, ist hier das ,Naturwesen“ Mensch, die ,von allen höheren Kräften und Werten abgelöste Menschengattung'“"34 Was verbindet das „Naturwesen Mensch“ zu einer kulturellen und humanen Gemeinschaft? Wovon kann ein Staat, bestehend aus einer bloßen Ansammlung von biopsycho-sozialen Individuen, ähnlich einer Nutztierhaltung, getragen sein? Wie kann ein gesellschaftlicher Zusammenhalt begründet werden, wenn es kein ideelles gemeinsames Band unter den Menschen gibt, keine von Gott legitimierte Herrschaft, keine Bindung an einen gemeinsamen Glauben, keine verbindende wesenhafte Natur des Menschen? Auf welcher Grundlage sollten sich Individuen, welche jeweils nur am eigenen Wohlbefinden (oder jenem ihrer Sippe) orientiert sind, zu einer sozialen Gemeinschaft mit gegenseitigen Verpflichtungen und gesetzlich erzwungener Solidarität als „öffentlichem Gut“ 35 zusammenschließen? Das ist das Grundproblem aller modernen (und d.h. naturalistischen) Gesellschaftstheorien, die eine Antwort nur auf Basis natürlicher Gegebenheiten zu finden imstande sind, da der Naturalismus, d. h. das Beherrschungswissen im Sinne der Wissensformen Max Schelers, ${ }^{36}$ der einzig verfügbare epistemologische Rahmen ist.

Für die Frage nach der Legitimation gibt es im Rahmen der modernen Wissensform und naturalistischen staatlichen Ordnung nur zwei mögliche Wirklichkeitsbereiche, die den Anspruch (oder den Anschein) des Absoluten aufweisen und damit gesellschaftlich die Voraussetzung der formalen „Unverfügbarstellung“37 (als Surrogat für Transzendenz) beanspruchen können:

a) das Kollektive bzw. Soziale (z. B. kontraktualistisch, über den Vertrag konstituiert) und

b) die Natur (über die „Naturalität der Mechanismen“38 wie jener des Wettbewerbs auf Märkten).

33 Delikostantis, Humanitarismus, 7.

34 Max Scheler, zit. n. Delikostantis, Humanitarismus, 51.

35 Vgl. Baurmann, Solidarität.

36 Scheler, Wissensformen, $203 \mathrm{f}$.

37 Vgl. Vorländer, Transzendenz.

38 Foucault, Sicherheit, 501. 
Die beiden Sphären des Kollektiven und der Natur werden für die modernen Staats- und Politikkonzeptionen die maßgebenden Instanzen für eine gerechte Gesellschaftsordnung in der Form einer Normativität, die jedenfalls auf natürlichen oder kollektiv vereinbarten Mechanismen beruhen muss. Den beiden Sphären entsprechen zwei komplementäre Formen von „Gerechtigkeitsmechanismen“:

a) die soziale Sorge basierend auf vereinbarten Gleichheits- und Solidaritätsdoktrinen und

b) die „naturgesetzlich“ entstehende Gerechtigkeit durch „natürliche“ Mechanismen wie Konkurrenz und Wettbewerb.

Den beiden Mechanismen der "gerechten“ gesellschaftlichen Ordnung entsprechen ebenso komplementäre biopolitische Steigerungstechniken:

a) die Förderung (Inklusion) der Förderungswürdigen (entsprechend dem faire vivre) und

b) die Ausscheidung (Exklusion) der Nicht-Förderungswürdigen (entsprechend dem laisser mourir).

Beide Techniken stehen im Zeichen der biopolitischen Lebenssteigerung, deren Kernaufgabe es ist, „das Lebende in einem Bereich von Wert und Nutzen zu organisieren“ 39 und die „ständige Erhöhung der Nützlichkeit [...] der Körper der Individuen und der Körper der Bevölkerungen" 40 durch die neuen Institutionen sicherzustellen. Denn die Anerkennung des Lebens als obersten Wert impliziert das „Wachsenwollen“, die Steigerung. 41

\section{3. „Beziehungen biologischen Typs“: Die Geburt der biopolitischen}

Steigerungsdispositive aus der hygienischen Bedrohung

Im biopolitischen Staat definieren sich die Verhältnisse zwischen den Menschen im Kern nicht auf der Ebene von Rechtsbeziehungen oder von Freundschaft und Feindschaft, sondern sie sind primär „Beziehungen biologischen Typs" (relations de type biologique), ${ }^{42}$ woraus die Bedeutung hygienischer Modelle für die gesellschaftliche Ordnung unmittelbar folgt. Es

39 Foucault, Wille, 139.

40 Foucault, Schriften, $25 \mathrm{f}$.

41 Vgl. Heidegger, Holzwege, $209 \mathrm{f}$.

42 Foucault, Société, 228. 
gibt davon zwei Hauptformen, die schon in der Formel vom „faire vivre et laisser mourir" anklingen:

a) die Relation des faire vivre, d.h. der "biopolitischen Sorge“ (als einer bio-psycho-sozialen Förderung) oder Solidarität (institutionell als Sozialisierung der allgemeinen Lebensrisiken) - man kann sie als den sozialbygienischen Typus bezeichnen - und

b) die Relation des laisser mourir, d. h. der Entsolidarisierung oder Konkurrenz (institutionell als Individualisierung der Lebensrisiken, z. B. durch Wettbewerb) - man könnte sie in historischer Anlehnung den rassenbygienischen Typus nennen.

Die beiden Formen begründen zwei wesentliche biopolitische Regierungstechniken: jene der biopolitischen Sorge gemäß einer Solidaritätsdoktrin, wie sie François Ewald in seiner Studie zum Vorsorgestaat (frz. L'État providence $)^{43}$ detailliert untersuchte, und jene des Staatsrassismus (racism d'État oder racism moderne), wie er von Foucault in der Vorlesung vom 17. März 1976 als Begriff und Konzept eingeführt wurde. ${ }^{44}$ Beiden Regierungstechniken ist gemeinsam, dass sie ihre Begründung in der Reaktion auf biologische Bedrohungen finden, welchen das Leben des Einzelnen oder der Bevölkerung ausgesetzt ist. Im ersten Fall ist es eine Beziehung der Art, dass die (hygienische oder biopsychosoziale) Sorge um den Anderen auch die eigene Sicherheit erhöht, das eigene Leben steigert, wie auch jenes der Gattung (Menschheit); es geht daher um Förderung und Solidarität im Sinne des faire vivre. Im zweiten Fall ist es genau umgekehrt: Es geht darum, „die biologische Gefahr zu beseitigen und die Gattung selbst oder die Rasse mit dieser Beseitigung direkt zu stärken“, denn, so die implizite Logik, ,je mehr anomale Individuen vernichtet werden, desto weniger Degenerierte gibt es in Bezug auf die Gattung, desto besser werde ich - nicht als Individuum, sondern als Gattung - leben, stark sein, kraftvoll sein und gedeihen"45. Die Darstellung dieses biopolitischen Rassismus folgt in einem zweiten Schritt. Zunächst wird das Solidaritätsmodell nach der Analyse von François Ewald, einem Mitarbeiter Foucaults am Collège de France, skizziert.

43 Ewald, Vorsorgestaat.

44 “Ce qui a inscrit le racisme dans les mécanismes de l'État, c'est bien l'émergence de ce bio-pouvoir"; Foucault, Société, 213 u. 227 f.

45 Foucault, Société, 228. 


\section{1 "Lebenmachen": Die biopolitische Sorge infolge pandemischer Bedrohungen, z. B. durch Vergemeinschaftung ökonomischer Risiken unter dem Banner der Solidarität}

Der säkulare Wohlfahrtsstaat als Institutionalisierung einer auch biopolitisch grundierten Solidarität stand also seit seiner ersten Ausbildung im 19. Jahrhundert vor dem Problem, die moralische und gesetzliche Verpflichtung des Einzelnen zu Solidaritätsleistungen (also Zwangssolidarität) zu begründen, und dies ohne Rückgriff auf die im Verschwinden begriffenen religiös verwurzelten Moralvorstellungen, wie z. B. jene der christlich motivierten Nächstenliebe. Die Frage war also: Wie lassen sich ein Mutualitätsprinzip bzw. soziale Rechte ohne metaphysische Begriffe und Normen begründen? Nach der Analyse Ewalds geschah dies unter Rückgriff auf Modellvorstellungen aus dem Gebiet der Öffentlichen Hygiene. Louis Pasteurs Entdeckungen zur Infektionsepidemiologie der ansteckenden Krankheiten dienten als Modell für die Verflochtenheit des „sozialen Übels“, das sich immer als Folge der gesellschaftlichen Umstände erweist und alle Mitglieder einer Gesellschaft zu gegenseitiger Solidarität und Vorsorge zwinge. Durch die hygienisch begründeten Solidaritätsdoktrinen, so jene des politisch einflussreichen französischen Sozialisten und Friedensnobelpreisträgers Léon Bourgeois, war eine Überwindung der liberalen Rechtsauffassung im Sinne der Ziele der Biopolitik möglich geworden. Als Modell fungierte für Bourgeois die ansteckende Krankheit, speziell die verbreitete Tuberkulose als die „Solidaritätskrankheit par excellence“, ${ }^{46}$ und die zentrale Gestalt war für ihn Pasteur:

$[W]$ enn man $[\ldots]$ das zum Ausdruck bringen wollte, was der gesunde, kräftige und wohlgestaltete Mensch von morgen sein soll, dann muss im Mittelpunkt dieses Tempels des Menschen eine Statue errichtet werden, die Statue Pasteurs. Ihm verdanken wir, dass die Vorstellungen von einer neuen Menschheit Gestalt annehmen [...]. Er war es, der die tiefgreifende, wechselseitige Abhängigkeit alles Lebenden, aller Lebewesen, endgültig bewies $[\ldots] .47$

46 Ewald, Vorsorgestaat, 467.

47 L. Bourgeois, zit. n. Ewald, Vorsorgestaat, 465. 
Und im Hinblick auf Pasteurs Mikrobenlehre der „zahllosen, unendlich kleinen Wesen" schreibt er:

Er hat uns bewiesen, dass jeder von uns für die anderen Lebenden zu einem Herd des Todes werden kann und dass wir infolgedessen die Pflicht haben, diese tödlichen Keime zu vernichten, um sowohl unser eigenes Leben als auch das Leben aller anderen zu schützen und zu bewahren. 48

So ist das Kunststück gelungen: Die naturwissenschaftliche Lehre von der „sozialen Natur“ des Übels in Form der hygienischen gegenseitigen Bedrohung erlaubte die Ableitung einer Sozialmoral aus dem natürlichen Sein des menschlichen Kollektivs. ${ }^{49}$ "Die Verfolgung meines eigenen Wohls verpflichtet mich dazu, auch das Wohl der anderen zu wollen“, und wie Ewald weiter ausführt: „Kein Gott, nichts außerhalb eines jeden von uns ist mehr nötig, um uns dazu zu verpflichten, im eigenen Interesse das Wohl der anderen zu wollen." 50

Bei den neuen Formen der Solidarität und Humanität - und das ist paradigmatisch die zweite Seite der "Sorge“ in der biopolitischen Form des faire vivre - geht es "nicht mehr um die Unterstützung für einen besonders zerbrechlichen - verwirrten und verstörenden - Randbereich der Bevölkerung, sondern darum, wie sich das Gesundheitsniveau des Sozialkörpers in seiner Gesamtheit anheben lässt [...]. Der Imperativ der Gesundheit: Pflicht eines jeden und allgemeines Ziel. "51 Foucault bringt hier ein wesentliches Charakteristikum der im 18. Jahrhundert in Erscheinung tretenden modernen Solidarität und ihres Humanitätskonzepts auf den Punkt: Es geht, auch wenn diese Rhetorik oft gepflegt wird, in erster Linie nicht um eine auf jene vulnerablen Bevölkerungsteile beschränkte Hilfe in der Not, die dieser wirklich bedürften, sondern um die Wohlfahrt und Förderung der menschlichen Gattung, begleitet von der Forderung einer materi-

48 L. Bourgeois, zit. n. Ewald, Vorsorgestaat, 465.

49 Vgl. die Ursprünge der Sozialhygiene in Deutschland: „Die erste Monographie im deutschen Sprachgebiet über Soziale Hygiene schrieb K. GEORG im Jahr 1890 [...]. GEORG bedeutete Sozialhygiene nichts weiter als die Anwendung allgemeinhygienischer Erkenntnisse auf die Lebensverhältnisse des Proletariats. Eigentlicher Beweggrund für diese ,soziale ${ }^{\natural}$ Auffassung war einerseits die Erkenntnis, dass das Proletariat die gesundheitlich labilste Bevölkerungsklasse ist, und dass andererseits jede Klasse von der anderen auf Gedeih und Verderb abhängt. Es war also letzten Endes der Egoismus der besitzenden Klasse die Triebfeder für eine ,Soziale Hygiene“ im Sinne GEORGS“; Thissen, Entwicklung, 60.

50 Ewald, Vorsorgestaat, 467.

51 Foucault, Schriften, 23. 
ell konnotierten „allgemeinen Menschenliebe“ und ihrer Realisierung über öffentliche Institutionen. Sowohl in dieser Orientierung auf die Steigerung der allgemeinen Wohlfahrt als auch in der staatlichen (oder staatsnahen) Institutionalisierungsform unterscheidet sich die moderne Solidarität wesenhaft von einer Nächstenliebe und Caritas für Leidende und Bedürftige. ${ }^{52}$ In diesem Sinne kann Ewald sagen:

Im Vorsorgestaat erfüllt sich dieser Traum von einer ,Bio-Macht'. Er ist ein Staat, der weniger darauf achtet, die Freiheit eines jeden vor Angriffen zu bewahren [...], als sich der Art und Weise anzunehmen, in der der einzelne sein Leben plant. [...] Über Institutionen wie die Sozialversicherungen wird er das Leben der Bevölkerungen als solcher verwalten können, um sie besser vor sich selbst zu bewahren und ihr die Entfaltung der in ihr schlummernden Potentialitäten zu ermöglichen. ${ }^{53}$

Der Entfaltung der Biopolitik eröffnet sich mit den sozialen Rechten des Wohlfahrtsstaats ein neues, weites Feld der Institutionalisierung der Förderung der Lebenschancen und Kompetenzen der Individuen zu ihrem eigenen Besten. Der Preis dafür ist jedoch der Verlust der konzeptuellen Trennung von Recht und Moral. ${ }^{54}$ Diesen Traum (und den Preis für die Verschmelzung von Recht und Moral) konkretisierte Léon Bourgeois 1914 u. a. mit den folgenden Worten:

Unsere Aufgabe liegt in der Koordinierung aller Vorsorgemaßnahmen gegen alle sozialen Übel, und bereits die Tatsache, dass diese Übel soziale Auswirkungen haben, genügt, um der Gesellschaft die Verpflichtung aufzuerlegen, sie vorherzusehen, zu bekämpfen und auszumerzen. Zu diesem Zweck muss das Individuum von Geburt an in Obhut genommen und bis zu seinem Tod Schritt für Schritt begleitet werden; in allen Stadien seiner Existenz müssen alle Risiken, denen es sich aussetzen wird, vorhergesehen, und es muss im Vorhinein ihre umfassen-

52 Vgl. die Beschreibung der Humanität durch Paul Göhre: „Auch Humanität ward erst mit und durch den modernen Menschen geboren. Humanität unterscheidet sich wesenhaft von Nächstenliebe. Diese ist persönlich bedingt [...], jene ist unpersönlich (!), gilt der gesamten Gattung, ja allem Lebendigen, drängt auf Institutionen, nicht mehr, wie jene, auf Ausübung von Fall zu Fall und in beschränktem Kreise"; Göhre, Gott, 21.

53 Ewald, Vorsorgestaat, 488.

54 Zur Frage nach der durch Freiheitsrechte erzeugten Unfreiheit, auch in Bezug auf die sozialen Teilhaberechte, gibt Christoph Menke (Menke, Kritik, 293 f.) eine Analyse im Anschluss an Foucault. 
de Absicherung organisiert werden. Dies ist kein Werk der Wohltätigkeit, sondern ein Werk der Vorsorge, kein individuelles, sondern ein universelles Werk [...].55

Léon Bourgeois war mit diesen Ideen sozialer Verpflichtung und Solidarität zu jener Zeit nicht allein. Einige Jahre zuvor veröffentlichte der österreichische Soziologe Rudolf Goldscheid ein System der „Menschenökonomie“ 56 , das die „unerhörte Menschenvergeudung" beklagte, die eintrete, wenn das „Menschenmaterial“ nicht nach einer rationalen, d. h. wirtschaftlich optimalen Verwaltung und Sorge behandelt werde.

Goldscheid entwirft eine gleichermaßen sozialistische wie biopolitische Gouvernementalität, welche nicht dem Markt-, sondern dem Mutualitätsprinzip gehorcht und welche die ökonomische Rationalisierung des Lebens auf präventive Pflege, nachhaltige Nutzung und wechselseitige Absicherung der menschlichen Ressourcen aufbaut. Fortschritt und Solidarität sind ihre Maximen, Akkumulation des organischen Kapitals ist ihr Bewegungsprinzip. ${ }^{57}$

Wenn Goldscheid die „Menschenvergeudung“ beklagt, so ist es ein Angriff auf die liberale politische Rationalität, denn, wie Ewald zusammenfasst, „[d]er Vorsorgestaat wirft dem liberalen Staat vor, ein schlechter Verwerter

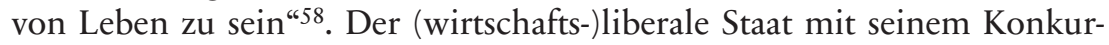
renzprinzip und Marktmechanismus realisiert jedoch die komplementäre biopolitische Steigerungstechnik der Elimination durch Wettbewerb, und auch diese folgt u. a. naturwissenschaftlich-hygienischen Doktrinen.

\section{2 "Sterbenlassen“: Biopolitischer Rassismus infolge von pandemischen Bedrohungen, z. B. durch Verzicht auf epidemiologisch indizierte Infektionsprophylaxe}

Wenn das oberste Ziel der biopolitischen Förderung und Sorge die Steigerung des Produktivitätsniveaus und des Gesundheitsniveaus des Sozialkörpers ist, was soll dann mit jenen geschehen, die nicht effizient förderbar sind oder sich der Sorge entziehen und damit in letzter Konsequenz das wirtschaftliche und gesundheitliche Niveau des Sozialkörpers, die allge-

55 L. Bourgeois, zit. n. Ewald, Vorsorgestaat, 531.

56 Vgl. Goldscheid, Entwicklungswerttheorie.

57 Bröckling, Hirten, 331.

58 Ewald, Vorsorgestaat, 488. 
meine Wohlfahrt, belasten und beeinträchtigen? Diese logische Folgefrage stellt sich unmittelbar nach Etablierung von biopolitischen Sorgetechniken, wie jenen in Form der Sozialhygiene, und unmittelbar entwickelt sich auch ab der zweiten Hälfte des 19. Jahrhunderts ein neuer Diskurs im Bereich der Medizin und Hygiene: die Ergänzung der Sozialhygiene durch die im deutschsprachigen Raum sogenannte Rassenhygiene (in den angelsächsischen Ländern als Eugenik bezeichnet).

Ein bedeutender Proponent der Kombination beider Techniken war der Inhaber des ersten Lehrstuhls für Sozialhygiene in Deutschland, der Arzt Alfred Grotjahn. Im Jahr 1912 erschien sein Hauptwerk Soziale Pathologie, in dem er sowohl sozialhygienischen Ideen von der sozialen Ätiologie der Krankheiten folgt als auch rassenhygienischen Ideen, sofern sie dem obersten Ziel der Herstellung einer gesunden Gesellschaft, bestehend aus „muskelstarken, breitschulterigen, organgesunden Starken und Rüstigen" ${ }^{59}$, dienen. Aufgrund von Krankheitsstatistiken hielt er ungefähr ein Drittel der Bevölkerung für gesundheitlich „minderwertig“ und die vorgeschlagenen Lösungen beruhten, seinen beiden komplementären Ansätzen entsprechend, auf der Unterscheidung von

a) umweltbedingten Krankheiten - es geht darum, gesellschaftliche Bedingungen zu bessern (Sozialhygiene) - und

b) anlagebedingten Krankheiten - rassenhygienische Maßnahmen, um „körperlich Minderwertige und Schwächlinge“ zu verhindern.

Als Lösung für die vorhandenen ,geistig Minderwertigen, die als Irre, Gewohnheitsverbrecher, unheilbare Trunkenbolde und überhaupt unverbesserliche Psychopathen ohnehin aus der Bevölkerung ausgeschieden werden müssen“, schlägt er die „planmäßige Ausmerzung durch Verwahrung und Zwangsunfruchtbarmachung“ vor, wofür die „dauernde Asylierung“ „ein humanes und wirksames Mittel“ sei. ${ }^{60}$

\section{3 „Sterbenmachen“: Die „Regeneration" der Bevölkerung durch ibre Auslieferung an Todesrisiken wie Pandemien}

Grotjahn charakterisiert paradigmatisch die biopolitische Doppelstrategie von Sorge, d. h. Förderung der Förderungswürdigen, bei gleichzeitiger Abtrennung der Nicht-Förderungswürdigen. Wenn die letztere Gruppe eine

59 Grotjahn, Hygiene, $185 \mathrm{f}$.

60 Grotjahn, Hygiene, 200. 
Last oder sogar biologische Gefahr für den Sozialkörper oder die Gattung darstellt, und darunter fallen insbesondere (unproduktive) chronisch Kranke und infektiöse Personen, so rechtfertigt die Logik der Betrachtungsweise ihre „Beseitigung“ im Sinne der Lebensförderung „aller“, gleichsam ihre Opferung für das Leben im Großen, um „die Gattung selbst oder die Rasse mit dieser Beseitigung direkt zu stärken"61. Das Dem-Tod-Ausliefern ist eine auf den ersten Blick paradoxe Steigerungstechnik im biopolitischen Staat, der sich doch der allgemeinen Wohlfahrt und einem Kult des Lebens verschreibt. Foucault verweist auf den epistemologischen Ursprung dieses politischen Diskurses in der biologischen Theorie des 19. Jahrhunderts: „[J]edes Mal, wenn es Konflikt, Tötung, Kampf, Todesrisiko gegeben hat, glaubte man sie buchstäblich in den Formen der Evolutionstheorie denken zu müssen." 62 Die Zurückführung der Politik, also der Beziehungen der Menschen untereinander im Großen, auf biologisch-hygienische Kategorien mündet in einem "hygienischen“ Verständnis von Krieg und von äußerer Bedrohung der eigenen Bevölkerung: Diese der biologischnatürlichen Todesgefahr auszusetzen, führe zu einer Regeneration bzw. Reinigung des eigenen biologischen Bestandes.

Aber darüber hinaus wird der Krieg - und das ist absolut neu - Ende des 19. Jahrhunderts als Möglichkeit erscheinen, nicht nur die eigene Rasse durch Beseitigung der gegnerischen Rasse zu stärken (entsprechend der Themen der Selektion und des Kampfes ums Dasein), sondern auch die eigene Rasse zu regenerieren [...]. Man geht bis zu dem Punkt, an dem die gesamte Bevölkerung dem Tod ausgeliefert wird. ${ }^{63}$

Hier wird das Wettbewerbsprinzip des (Wirtschafts-)Liberalismus ${ }^{64}$ naturalisiert und der kollektiven biologischen (Todes-)Gefahr eine biopolitische Funktion im Sinne der Lebenssteigerung durch den Mechanismus der „natürlichen Elimination“ der bio-psycho-sozial „Minderwertigen“ aus dem Sozialkörper gegeben. Das vorsätzliche (oder auch grob fahrlässige) Ausliefern der eigenen Bevölkerung an biologische Gefahren (danger biologique)

61 Foucault, Société, 228.

62 Foucault, Société, 229.

63 Foucault, Société, $229 \mathrm{f}$.

64 „Man kann sagen, dass die Devise des Liberalismus ist, ,gefährlich zu leben`. ,Gefährlich zu leben', das bedeutet, dass die Individuen fortwährend in eine Gefahrensituation gebracht werden oder dass sie vielmehr darauf konditioniert werden, ihre Situation, ihr Leben, ihre Gegenwart, ihre Zukunft usw. als Träger von Gefahren zu empfinden. Und dieser Anreiz der Gefahr ist, glaube ich, eine der wichtigsten Implikationen des Liberalismus“; Foucault, Geburt, 101. 
in Form von Kriegen oder epidemischen bzw. pandemischen Risiken kann so Teil einer Biopolitik der kollektiven Leistungs- und Lebenssteigerung sein. Gerade von der Last jener, die invalid sind oder aufgrund ihres Alters vermutlich „ohnehin nicht mehr lange zu leben haben"65, würde die Gesellschaft durch die reinigende Naturkraft tödlicher Pandemien befreit werden - so die Rationalität des Foucault'schen (Staats-)Rassismus des Krieges $^{66}$. Ein solches Ausliefern der Bevölkerung könnte im Verzicht auf infektionsepidemiologisch indizierte und verfügbare Schutzmaßnahmen der Seuchenprophylaxe (wie z. B. Quarantäne, social distancing etc.) unter Inkaufnahme der weiteren Verbreitung der Krankheit, insbesondere in vulnerablen Gruppen, bestehen.

\section{Résumé: Pandemien können die biopolitische Polarität von übersteigerter und feblender Solidarität verstärken}

Aus dem Gesagten ergibt sich als wesentliche Einsicht, dass im biopolitisch-gouvernemental geprägten Staatswesen die Unterscheidung zwischen Recht und Moral (heute meist als Ausdruck von „Werten“) tendenziell verschwindet. Die Auflösung dieser Differenz zerstört zuletzt sowohl das (vernunftbegründete) Recht als auch die Moral - beide verschmelzen im modernen Wohlfahrtsstaat zu einer „Sozialtechnik der Regierenden“, einem "Regierungsinstrument“, wie es François Ewald in seiner Studie Der Vorsorgestaat formuliert. ${ }^{67}$ Die Moral wird gewissermaßen verrechtlicht und in der Folge verstaatlicht, und reziprok verliert das Recht seine rationale Fundierung (z.B. in der Vernunft oder im Naturrecht) - es wird wertbegründet, ${ }^{68}$ u.a. im Namen einer "Solidarität“", mit den damit einhergehenden

65 Hier findet eine Abwertung der Lebenszeit hochaltriger Menschen einen Ausdruck, der im Zusammenhang mit den Maßnahmen zur Eindämmung der Covid-19-Pandemie gelegentlich in den Medien vorgebracht wurde.

66 „Vous avez là, en tout cas, un racism de la guerre, nouveau à la fin du XIXe siècle [...]"; Foucault, Société, 230.

67 Vgl. Ewald, Vorsorgestaat, 472: „Und so wie das Recht dazu tendiert, zu einer Lektion in Moral zu werden, wird all das, was moralisch gilt, auch seine rechtliche Gültigkeit erlangen“, denn wenn „das, was die Regierenden als nötig erachten, nicht auf natürlichem Wege geschieht, wird man es zu einer gesetzlichen Vorschrift machen. Das Recht verliert damit die Würde, die ihm aufgrund seiner Beziehung zu Natur und Vernunft zukam, es wird zu einer Sozialtechnik“.

68 Zur Problematik der Wertbegründung des Rechts vgl. Böckenförde, Kritik, 67 f. 
Problemlagen. ${ }^{69}$ Der biopolitische Normalisierungsstaat ist in einem bestimmten Sinne ein Moralstaat, beruhend auf dem Prinzip der Transparenz und Sichtbarkeit (vgl. den Panoptismus ${ }^{70}$ ) als einem konstitutiven sozialtechnologischen Dispositiv. Auch im liberalen Staat gilt, wie Foucault ausführt: „Mit einer Hand muss die Freiheit hergestellt werden, aber dieselbe Handlung impliziert, dass man mit der anderen Einschränkungen, Kontrollen, Zwänge, auf Drohungen gestützte Verpflichtungen usw. einführt". ${ }^{71}$

Die Sorge-Menschenökonomie des Vorsorge- bzw. Wohlfahrtsstaates sowie die liberale Wettbewerbs-Ökonomie der Humankapitaltheorie markieren zwei komplementäre Pole bzw. dialektische Gegensätze hinsichtlich ihrer Funktionsmechanismen und Strategien, treffen sich aber in ihrer biopolitischen Zielsetzung. ${ }^{72}$ Sie bilden - wie Ulrich Bröckling ausführt „zwei konträre Regierungsrationalitäten, zwischen denen die Biopolitiken des 20. und 21. Jahrhunderts changieren: Markt und Plan, unsichtbare oder sichtbare Hand, zentrale Steuerung oder Selbstorganisation - zwischen diesen Polen lassen sich die Versuche verorten, das menschliche Leben ökonomisch zu regieren "73. Solidarität und Entsolidarisierung, Fördern der Förderungswürdigen und Selektion der Nicht-(mehr-)Förderungswürdigen - zwischen diesen komplementären Polen agiert die biopolitische Rationalität. Beide Pole funktionieren, wie ausgeführt, zwar legitimiert von einer Logik der Abwehr hygienischer Gefahren, aber orientiert an gouvernementalen Zielsetzungen.

Gefährliche Epidemien oder Pandemien besitzen folglich das Potential, beide Pole zu stärken und die biopolitische Transformation der Gesell-

69 Zur Illustration sei dieses Zitat aus einem aktuellen Zeitungsartikel, Der Terror der Tugend (Martenstein, Terror), angeführt: „Unser Körper gehört nicht mehr uns alleine, wir haben unseren Körper offenbar vom Staat großzügig zur Verfügung gestellt bekommen, und der Staat verlangt, dass wir mit dieser Leihgabe pfleglich umgehen“..., Willkommen in der Tugendrepublik Deutschland! In der Tugendrepublik verschmelzen drei geistige und politische Tendenzen der letzten Jahre. Erstens die Tendenz zur Transparenz, die neue Geheimnislosigkeit. Zweitens die Idee, dass ein moralisch einwandfreies Leben des Individuums staatlich durchsetzbar sein könnte. Drittens der Gedanke der möglichst vollständigen Risikovermeidung, wobei die Entscheidung über individuelle Risiken nicht etwa im Ermessen des Individuums steht. Die Entscheidung über Risiken ist vielmehr Sache des Staates, der dabei von einem Heer aufmerksamer Hobbydetektive und emsiger Leserreporter unterstützt wird.“

70 Foucault, Überwachen, $251 \mathrm{f}$.

71 Foucault, Geburt, 98.

72 Stronegger, Institutionalisierung, 39.

73 Bröckling, Hirten, 331. 
schaft und die von Foucault beschriebene Gouvernementalisierung des modernen Staates zu intensivieren, indem Maßnahmen gesetzt werden, die über infektionsepidemiologisch indizierte Schutzmaßnahmen mit dem Ziel der Eindämmung der epidemischen Krankheitsverbreitung klar hinausgehen. $\mathrm{Zu}$ diesen potentiell biopolitisch motivierten Maßnahmen gehört im Pol der „biopolitischen Sorge“ ein infektionsprophylaktisch nicht indiziertes Ausmaß an Erfassung persönlicher Risikodaten (im Sinne der Pastoralmacht) oder eine Vergemeinschaftung ökonomischer Risiken im Namen einer Solidarität, die in keinem sachlichen Verhältnis zur epidemiologischen Gefahrenabwehr steht. Im Pol des „biopolitischen Rassismus" befindet sich konträr dazu beispielsweise der weitgehende Verzicht auf infektionsepidemiologisch indizierte und verfügbare Schutzmaßnahmen der Seuchenprophylaxe (wie z.B. Quarantäne, social distancing etc.) unter Inkaufnahme der weiteren Verbreitung der Krankheit und der daraus folgenden gesundheitlichen Gefährdung zusätzlicher Bevölkerungsteile.

Die politische Alternativlosigkeit dieser Transformation von historischer Dimension bleibt aber fragwürdig, denn die Verabsolutierung des sozialeudämonistischen Denkens, das im Zeichen einer naturalistischen Selbstauslegung des Menschen steht, d.h. die Ethisierung des natürlichen Wohlergehens, gefährdet im Gegenzug die unbedingte Anerkennung der Menschenwürde. So legt Delikostantis in einer Untersuchung des modernen Humanitarismus eine elementare Wahrheit dar: „Vom naturalistisch bestimmten Glück des Menschen als oberstem ethischen Maßstab aus gedacht, kann die Würde des Menschen nicht angemessen expliziert werden"74, da seine Rechtssubjektivität als nun tatsächlich alternativlose Basis seiner Würde ${ }^{75}$ Gefahr läuft, lebensweltlich ohne Anerkennung zu bleiben. Oder mit den Worten Foucaults: „Desormais, la sécurité est au-dessus des lois.“ Die optimierte Lebenssicherung und Lebenssteigerung ist mit dem Konzept individueller Freiheit in letzter Konsequenz realweltlich inkommensurabel. An dieser für den zeitgenössischen Menschen wohl schwer nachvollziehbaren Feststellung zeichnet sich bereits die Ambivalenz bzw. Dialektik der biopolitischen Transformation ab, in der sich die Industriegesellschaften seit ihrer Genese befinden: „Eine Entwicklung in

74 Delikostantis, Humanitarismus, 7.

75 Vgl. die Studie zur Grundlegung der Menschenwürde von Walter Schweidler (Schweidler, Menschenwürde). 
der modernen Moral, die sich als Förderung der Humanität begreift und Geltung verschaffen will, erscheint als Gefährdung der Humanität." ${ }^{\text {“76 }}$

\section{Literatur}

Baurmann, Michael: Solidarität als soziale Norm und als Norm der Verfassung, in: Kurt Bayertz (Hg.): Solidarität, Frankfurt am Main: Suhrkamp 1998, 345-388.

Böckenförde, Ernst-Wolfgang: Die Entstehung des Staates als Vorgang der Säkularisation, in: Ders.: Recht, Staat, Freiheit. Studien zur Rechtsphilosophie, Staatstheorie und Verfassungsgeschichte, Frankfurt am Main: 1991, 42 [1967].

Böckenförde, Ernst-Wolfgang: Zur Kritik der Wertbegründung des Rechts, in: Ders.: Recht, Staat, Freiheit. Studien zur Rechtsphilosophie, Staatstheorie und Verfassungsgeschichte, Frankfurt am Main: 1991, 67-91.

Bröckling, Ulrich: Gute Hirten führen sanft. Über Menschenregierungskünste, Berlin: Suhrkamp 2017.

Delikostantis, Konstantinos: Der moderne Humanitarismus. Zur Bestimmung und Kritik einer zeitgenössischen Auslegung der Humanitätsidee, Mainz: Grünewald 1982.

Ewald, François: Der Vorsorgestaat, Frankfurt am Main: Suhrkamp 1993 [frz. 1986].

Foucault, Michel: Überwachen und Strafen. Die Geburt des Gefängnisses, Frankfurt am Main: Suhrkamp ${ }^{14} 2016$ [1975].

Foucault, Michel: Die Geburt der Biopolitik. Geschichte der Gouvernementalität II. Vorlesungen am Collège de France 1978-1979, Frankfurt am Main: Suhrkamp 2004.

Foucault, Michel: Il faut défendre la société. Cours au Collège de France (19751976), Paris: Gallimard 1997.

Foucault, Michel: Dits et écrits 1954-1988, tome II: 1976-1988, Paris: Gallimard 2001.

Foucault, Michel: Schriften in vier Bänden. Dits et écrits, Bd. III: 1976-1979, Frankfurt am Main: Suhrkamp 2003.

Foucault, Michel: Sicherheit, Territorium, Bevölkerung. Geschichte der Gouvernementalität I. Vorlesung am Collège de France 1977-1978, Frankfurt am Main: Suhrkamp ${ }^{5} 2017$.

Foucault, Michel: Der Wille zum Wissen. Sexualität und Wahrheit 1, Frankfurt am Main: Suhrkamp ${ }^{14} 1977$.

Göhre, Paul: Der unbekannte Gott, Leipzig: Grunow 1919.

Goldscheid, Rudolf: Entwicklungswerttheorie, Entwicklungsökonomie, Menschenökonomie. Eine Programmschrift. Leipzig: Klinkhardt 1908.

76 Delikostantis, Humanitarismus, 7. 
Grotjahn, Alfred: Die Hygiene der menschlichen Fortpflanzung. Versuch einer praktischen Eugenik, Berlin: Urban \& Schwarzenberg 1926.

Heidegger, Martin: Holzwege, Frankfurt am Main: Klostermann 2015.

Martenstein, Harald: Der Terror der Tugend, in: http://www.zeit.de/2012/24/DOSTugend [12.05.2020].

Mau, Steffen: Das metrische Wir. Über die Quantifizierung des Sozialen, Berlin: Suhrkamp 2017.

Menke, Christoph: Kritik der Rechte, Frankfurt am Main: Suhrkamp 2015.

Nussbaum, Martha: Creating capabilities. The human development approach, Cambridge: Harvard University Press 2011.

Scheler, Max: Die Wissensformen und die Gesellschaft, Bern: Francke 1960.

Schulz, Walter: Philosophie in der veränderten Welt, Pfullingen: Neske 1972.

Schweidler, Walter: Über Menschenwürde. Der Ursprung der Person und die Kultur des Lebens, Wiesbaden: Springer 2012.

Stronegger, Willibald J.: Die Legitimität von Tötungshandlungen im modernen Staat - Eine biopolitische Perspektive, in: Imago Hominis 23 (2016), 35-45.

Stronegger, Willibald J.: „Faire vivre“. Die Institutionalisierung des Lebens in der Moderne und der Tod, in: Stronegger, Willibald J./Attems, Kristin (Hg.): Mensch und Endlichkeit. Die Institutionalisierung des Lebensendes zwischen Wissenschaft und Lebenswelt, Baden-Baden: Nomos 2018 (= Bioethik in Wissenschaft und Gesellschaft 5), 23-53.

Thissen, Rudolf: Die Entwicklung der Terminologie auf dem Gebiet der Sozialhygiene und Sozialmedizin im deutschen Sprachgebiet bis 1930, Wiesbaden: Springer 1969.

Vorländer, Hans (Hg.): Transzendenz und die Konstitution von Ordnungen, Berlin: De Gruyter 2013. 
